\title{
InP Quantum Dot Mode-Locked Lasers and Materials Studies
}

\author{
Zhibo $\mathrm{Li}^{1}$, Samuel Shutts ${ }^{1}$, Craig P. Allford ${ }^{1}$, Andrey B. Krysa ${ }^{2}$, Peter M. Smowton ${ }^{1}$ \\ ${ }^{1}$ EPSRC Future Compound Semiconductor Manufacturing Hub, Cardiff University, Cardiff, CF24 3AA \\ ${ }^{2}$ EPSRC National Centre for III-V Technologies, University of Sheffield, Sheffield, S1 3JD \\ LiZ74@cardiff.ac.uk
}

\begin{abstract}
InP/GaInP quantum dot laser structures exhibiting broad optical gain spectra suitable for modelocking have been demonstrated. Two-section narrow ridge passive mode-locked lasers were fabricated from this material. Mode-locking conditions have been investigated for devices with different cavity lengths, with maximum frequency of $15.21 \mathrm{GHz}$.
\end{abstract}

Key Words-InP Quantum Dots, Mode-Locked Lasers, Optical gain and absorption

\section{INTRODUCTION}

Semiconductor monolithic mode-locked lasers (MLL) emitting in the visible to near infrared region are required for small footprint, low cost and on chip alternatives to the Ti: Sapphire laser and specifically for applications in twophoton fluorescent imaging. This imaging technique requires fluorescent molecules to simultaneously absorb two photons with half the energy of the excited transition, and then achieve fluorescence emission. This technique offers deeper tissue penetration depth, higher resolution with reduced background signal interference and less photobleaching than the single photon fluorescent imaging. Quantum dot materials with their broad optical gain features are promising to achieve ultrashort pulses via mode-locking [1]. Here we demonstrate mode-locking in a monolithic structure using InP quantum dots (QD) as the active region in an epitaxial structure that can be extended to cover the $630-780 \mathrm{~nm}$ range $[2,3]$ and that can be integrated with biomedical diagnostic configurations [4].

\section{MATERIAL AND DEVICE}

Figure 1 gives the laser structure grown on $n-G a A s(100)$ substrates oriented $10^{\circ}$ off toward $\langle 111\rangle$ direction by MOCVD. Self-assembled InP QDs were covered by lattice-matched GaInP quantum wells to form each DWELL layer which was separated by AlGaInP barriers. $\mathrm{AlGaInP}$ forms the top and bottom waveguide and cladding layers, and a heavily p-doped GaAs cap was grown for the top contact.

The sample material was firstly patterned with $2 \mu \mathrm{m}$ wide shallow-etched ridges by electron beam lithography and inductively coupled plasma (ICP) etching, followed by a planarization step using Benzocyclobutene $(\mathrm{BCB})$ and etch-backed to the ridge-top using reactive ion etching. $\mathrm{Cr} / \mathrm{Au}$ were deposited as p-contact metals, with a gap in the contact between gain and saturable absorber (SA) sections of $20 \mu \mathrm{m}$. Sufficient electrical isolation was achieved by wet-etching the exposed p-GaAs layer between the contacts.. After lapping to $100 \mu \mathrm{m}$, the AuGe/Ni/Au n-type contact metals were deposited on the substrate side and contacts were annealed. Broad-area multi-segmented contact devices with $100 \mu \mathrm{m}$ mesa width and $50 \mu \mathrm{m}$ pcontact width were fabricated for optical gain measurements.

\begin{tabular}{|l|}
\hline $300 \mathrm{~nm}$ GaAs contact $(\mathrm{p}+)$ \\
\hline $1050 \mathrm{~nm}$ AlGalnP cladding $(\mathrm{p})$ \\
\hline $180 \mathrm{~nm}$ AlGalnP cladding (un-doped) \\
\hline $16 \mathrm{~nm}$ AlGalnP quantum barrier $\times 4$ (un-doped) \\
\hline $8 \mathrm{~nm}$ GaInP quantum well $\times 5$ ( un-doped ) \\
\hline $6 \mathrm{~nm}$ InP quantum dot $\times 5$ (un-doped) \\
\hline $100 \mathrm{~nm}$ AlGalnP cladding (un-doped) \\
\hline $1050 \mathrm{~nm}$ AlGaInP cladding $(\mathrm{n})$ \\
\hline $500 \mathrm{~nm}$ GaAs buffer layer $(\mathrm{n})$ \\
\hline
\end{tabular}

Figure 1, InP/GaInP DWELL laser structure.

\section{RESULTS AND DISCUSSIONS}

Figure 2 shows the modal absorption and gain spectra, determined using the segmented contact method. Gain was measured for increasing injection current, up to a peak value of $10.8 \mathrm{~cm}^{-1}$ and the internal optical loss, taken at wavelengths beyond the band edge, was found to be $\sim 1.2$ $\mathrm{cm}^{-1}$.. At high current the optical gain spectra are relatively flat topped and reaching a width of approximately $50 \mathrm{~nm}$ (red curve).

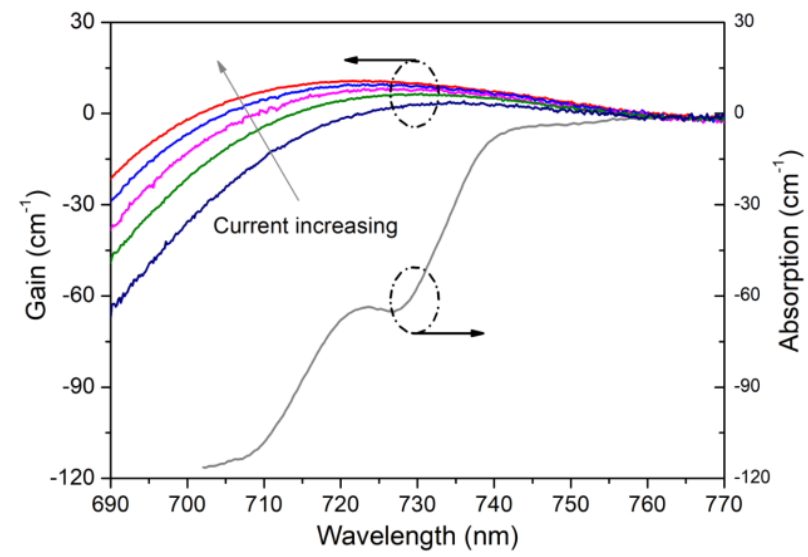

Figure 2, Net modal gain and absorption of InP QD materials.

Figure 3 plots the optical gain values and peak gain positions respective to the injection current density level. The peak wavelength blue-shifted from $734 \mathrm{~nm}$ to $722 \mathrm{~nm}$ with increasing injection, which is largely due to statefilling of the inhomogeneous distribution of energy states 
associated with a small and large ensemble of dot sizes. At higher injection currents recombination from the excited dot states at shorter wavelengths starts to play a role.

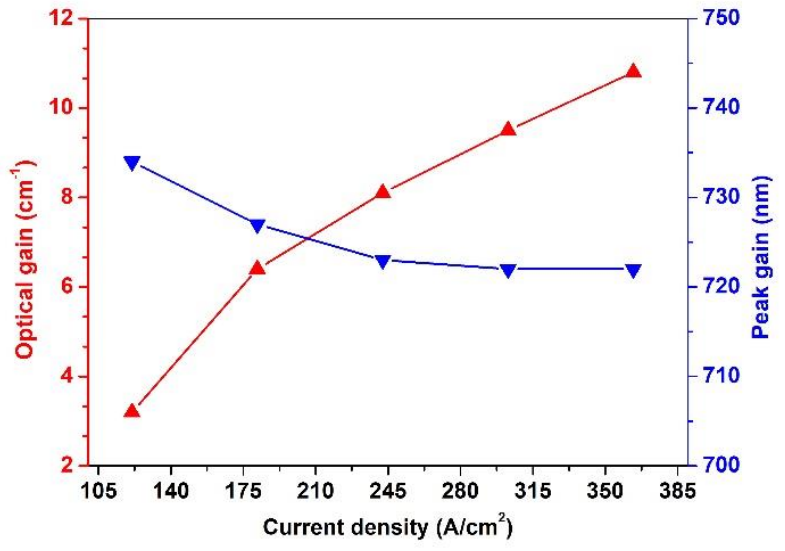

Figure 3, Optical gain magnitude and peak gain spectral position with respect to injection current density level

We utilized the optical gain measurements to design cavity lengths and length ratio of absorber and gain sections using the approach described in [5]. MLLs with 20\% ratio of SA length to the total length and with 3 different cavity lengths were fabricated. No facet coatings were applied to these devices. Figure 4 shows the light-current (L-I) measurements of a $3 \mathrm{~mm}$ long MLL driven by a pulsed current source $(1 \mu \mathrm{s}$ pulse width and $5 \mathrm{kHz}$ repetition frequency). When driving both sections in forward bias, the ridge laser gave a threshold current at $8 \mathrm{~mA}$. For samples where the absorber section was floating or reverse biased, a kink characteristic of saturable absorption was observed to a greater or lesser degree in the different devices.

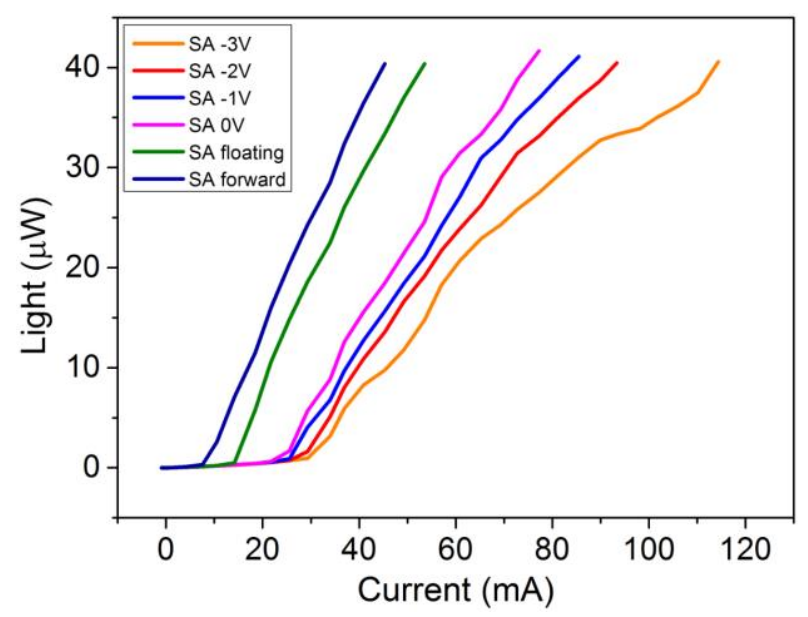

Figure 4, L-I features of a 3 mm length MLL with $20 \%$

SA section ratio under different drive conditions.

To measure the mode-locked pulse repetition frequency, a constant current source was used. The front facet emission was coupled into a fast-photodetector connected to an electrical spectrum analyzer. Figure 5 gives the repetition frequency of $3 \mathrm{~mm}$ long MLLs, with the inset showing the lasing spectra during mode-locking. The measured repetition frequency was $12.5 \mathrm{GHz}$ with a fundamental RF signal linewidth of $480 \mathrm{kHz}$, corresponds to cavity length of $3 \mathrm{~mm}$ as expected. Table 1 lists the mode-locking conditions of devices with cavity lengths of $2 \mathrm{~mm}, 3 \mathrm{~mm}$ and $3.5 \mathrm{~mm}$.

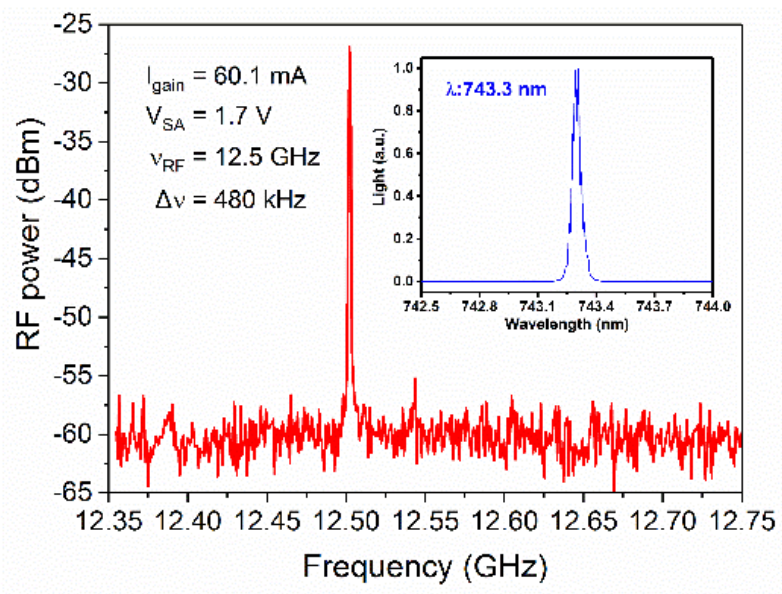

Figure 5, RF signals obtained from electrical spectrum analyzer.

\begin{tabular}{|c|c|c|c|c|}
\hline$L_{\text {cavity }}$ & $F_{\text {rep }}$ & $I_{\text {gain }}$ & $V_{S A}$ & RF linewidth \\
\hline $2 \mathrm{~mm}$ & $15.21 \mathrm{GHz}$ & $38 \mathrm{~mA}$ & $1 \mathrm{~V}$ & $380 \mathrm{kHz}$ \\
\hline $3 \mathrm{~mm}$ & $12.5 \mathrm{GHz}$ & $60 \mathrm{~mA}$ & $1.7 \mathrm{~V}$ & $480 \mathrm{kHz}$ \\
\hline $3.5 \mathrm{~mm}$ & $10.8 \mathrm{GHz}$ & $88 \mathrm{~mA}$ & $1 \mathrm{~V}$ & - \\
\hline
\end{tabular}

Table 1, Mode-locking conditions of devices with different cavity lengths.

\section{SUMMARY}

In summary, we studied the optical gain properties of InP/GaInP QD laser structure and demonstrated the first mode-locked lasers on this material.

This research is supported by the UK Engineering Physical Sciences Research Council (EPSRC) under grant number $\mathrm{EP} / \mathrm{P} 006973 / 1$

\section{REFERENCES}

[1] E. U. Rafailov, M. A. Cataluna, W. Sibbett, "Mode-locked quantum-dot lasers", Nature Photonics, 1, 395-401 (2007)

[2] I. Karomi, P.M. Smowton, S. Shutts, A.B. Krysa, R. Beanland, "InAsP quantum dot lasers grown by MOVPE", Optics Express, 23 (21), pp 27282-27291, (2015)

[3] S. Shutts, S.N. Elliott, P.M. Smowton, A.B. Krysa, "Exploring the wavelength range of InP/AIGaInP QDs and application to dual-state lasing", Semiconductor Science and Technology, Vol. 30 (4), 044002 (2015)

[4] R. Thomas, A. Harrison, D. Barrow, P.M. Smowton, "Photonic integration platform with pump free microfluidics" Optics Express, 25, pp.23634-23644 (2017)

[5] J. K. Mee, R. Raghunathan, J. B. Wright, L. F. Lester, "Device geometry considerations for ridge waveguide quantum dot mode-locked lasers," Journal of Physics D: Applied Physics, 47, 233001 (2014). 